\title{
Krüppel-Like Factor 7 is a Marker of Aggressive Gastric Cancer and Poor Prognosis
}

\author{
Zhonghua Jiang ${ }^{\mathrm{a}}$ Tingting Yu ${ }^{\mathrm{a}}$ Zhining Fan ${ }^{\mathrm{b}} \quad$ Hongmei Yang $^{\mathrm{a}} \quad$ Xin Lin ${ }^{\mathrm{c}}$ \\ aDepartment of Gastroenterology, the First People's Hospital of Yancheng, Yancheng, bepartment of \\ Digestive Endoscopy, the First Affiliated Hospital of Nanjing Medical University, Nanjing, 'Department \\ of Digestive Endoscopy, the First People's Hospital of Wujiang District, Suzhou, China
}

\section{Key Words}

Gastric cancer • Krüppel-like factor $7 \cdot$ Survival analysis $•$ Progression

\begin{abstract}
Background/Aims: Krüppel-like factor (KLF) 7 protein is a member of the KLF transcription factor family, which plays important roles in regulating the expression of genes involved in cell growth, proliferation, differentiation and metabolism. However, the role of KLF7 in gastric cancer (GC) is unknown. The aim of this study is to explore the role of KLF7 in GC and its correlation with clinicopathological characteristics and prognosis of GC patients. Methods: We first systematically evaluated dysregulation of the KLF family in The Cancer Genome Atlas (TCGA) GC database. Then, 252 patients who underwent surgery for GC were enrolled to validate the results from the TCGA. Functional studies were also used to explore the role of KLF7 in GC. Results: In the TCGA database, we found that KLF7 was an independent predictor for survival by both univariate and multivariate analysis $(P<0.05)$. In a validation cohort, KLF7 expression was significantly increased in GC tissues compared with adjacent normal controls $(P=0.013)$. High KLF7 expression correlated with inferior prognostic factors, such as $T$ stage $(P=0.022), \mathrm{N}$ stage $(P=0.005)$ and lymphovascular invasion $(P=0.009)$. Furthermore, we observed a strong negative correlation between KLF7 expression and 5-year overall survival and disease-free survival in GC patients $(P<0.05)$. Moreover, our in vitro studies showed a notable decrease in migration in KLF7 knockdown cells. Conclusion: KLF7 has an important role in GC progression, as it inhibits GC cell migration and may serve as a prognostic marker.
\end{abstract}

\section{Introduction}

(C) 2017 The Author(s)

Published by S. Karger AG, Basel

Although the incidence of gastric cancer (GC) is decreasing worldwide, it is still relatively high in eastern Asia, particularly in China and Japan [1]. In China, GC is predicted to rank as the second most common cancer, with 0.3 million deaths and 0.4 million new cases reported Z. Jiang and T. Yu contributed equally to this work.

\begin{tabular}{ll}
\hline Zhonghua Jiang, MD, & Department of Gastroenterology, the First People's Hospital of Yancheng. \\
and Hongmei Yang, MD & No. 166, West Yulong Road, Yancheng (China) \\
& E-Mail jiangzhonghua1982@163.com/196206yhm@163.com
\end{tabular}


each year [2]. Although significant progress has been made in the diagnosis and treatment of GC patients, complete resection remains the only treatment option that can lead to a cure for GC. However, a considerable proportion of patients will suffer recurrences and metastases after surgery. Moreover, one-third of GC patients have already reached late stage with distant metastasis at the time of diagnosis and have lost the option of surgical resection $[1,3]$. To increase the efficacy of GC therapy, it is important to identify candidate genes that are essential in cancer cell progression and metastasis, which could facilitate tumor detection and recurrence diagnosis at high sensitivity and specificity rates [4].

The Krüppel-like factor (KLF) family is a group of highly conserved zinc finger transcription factors, which are critical regulators of essential biological cellular processes, including proliferation, differentiation, apoptosis, and migration [5-8]. These proteins regulate gene expression by binding to GC rich sequences of gene promoters, the GC/GT boxes [9]. Recent attention has focused on the role of aberrant expression of the KLF family in cancer. For example, KLF8 activates the transcription of C-X-C cytokine receptor type 4 to promote breast cancer cell invasion, transendothelial migration and metastasis [10]. Loss of KLF4 and consequential downregulation of Smad7 exacerbate oncogenic TGF- $\beta$ signaling in and promote the progression of hepatocellular carcinoma [11]. KLF5 promotes cell migration by up-regulating FYN in bladder cancer cells [12]. However, the relationship between KLF family expression and GC has not been fully investigated.

In the present study, we first systematically study the KLF family members in The Cancer Genome Atlas (TCGA) RNA-sequence database to find new biomarkers for GC in terms of survival. Then, we validate the results in a cohort of in-house patients and in vitro experiments.

\section{Materials and Methods}

\section{Patients}

The TCGA gene expression database was used for primary study. TCGA (http://cancergenome.nih. gov/) is a project to catalogue genetic expressions or mutations responsible for cancer by bioinformatics methods and genome sequencing while the cBioPortal (http://www.cbioportal.org/index.do) is an exploratory analysis tool for exploring datasets from TCGA to find pathways of interest in one or more cancer types [13, 14]. Sequencing of the dataset was based on the Illumina HiSeq platform [15]. Inclusion criteria were as follows: patients with no pretreatment, with fully characterized tumors and intact overall survival (OS) information. Follow-up was completed on Dec 21, 2014. Expression data of each KLF family were standardized, and the standardization between different samples was performed by the Limma package of R software [16].

The validation cohort consists of 252 patients with histologically confirmed GC. All patients underwent radical resection between January 1, 2003 and December 31, 2010. No patients had preoperative therapies; no patients had evidence of metastasis at the time of diagnosis. Demographic and clinical characteristics, such as age, sex, age at initial diagnosis, and stage at diagnosis (tumor, node, metastasis [TNM] classification), were obtained from electronic records and are summarized in Table 1.

This study received Institutional Review Board approval from the No. 1 People's Hospital of Yancheng. Written informed consent was obtained from all subjects. The methods were performed in accordance with the approved guidelines.

\section{Cell culture}

The human GC cell lines AGS and MGC803 were originally purchased from the American Type Culture Collection. Cells were cultured in DMEM (Corning, Cellgro) plus 10\% fetal bovine serum (FBS) (Gibco), 100 $\mathrm{U} / \mathrm{mL}$ penicillin G, and $100 \mathrm{mg} / \mathrm{mL}$ streptomycin (Corning, Cellgro). All cells were cultured in a $37^{\circ} \mathrm{C}, 5 \%$ $\mathrm{CO} 2$ incubator.

Stable transfection of colon cancer cells

Biologically active short hairpin RNAs (shRNA) were generated using the lentiviral expression vector pLKO.1-puro. The shRNA target sequence for human KLF7 was 5'-CTTGAATTGGAACGCTACCTA-3'. PLKO.1- 


\section{Cellular Physiology Cell Physiol Biochem 2017;43:1090-1099

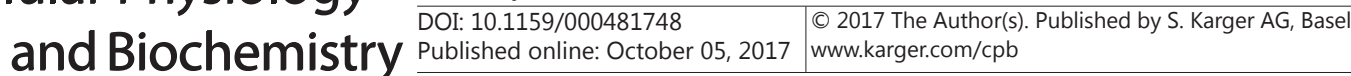 \\ Jiang et al.: KLF7 in Gastric Cancer}

scramble shRNA with limited homology to any known sequences in the human genome was used as a negative control. AGS and MGC803 cells were transfected with the pLKO.1-shKLF7 expression vector or pLKO.1-scramble. The stably transfected cells were isolated using puromycin selection to obtain stable KLF7 knockdown cells.

\section{Cell proliferation assays}

A cell proliferation reagent kit (CCK8, Dojindo, Japan) was used to assess cell proliferation. Transfected cells were plated in each well of a 96-well plate and assessed every $24 \mathrm{~h}$ according to the manufacturer's instructions. The cell viability of different groups at each time point was compared.

\section{Cell migration and invasion assay}

The migration and invasion ability of AGS and MGC803 cells after different transfections were measured by a transwell assay (without or with Matrigel). Approximately $10^{5}$ cells were seeded on the upper chamber of the transwell with $200 \mu \mathrm{l}$ serum-free growth medium $\left(10^{5}\right.$ cells per well of $8.0 \mu \mathrm{m}$ Pore Polycarbonate Membrane Insert). Complete medium containing 10\% FBS was added to the lower chamber as a chemo-attractant. After 48 $\mathrm{h}$ of incubation at $37{ }^{\circ} \mathrm{C}$, non-migratory cells on the upper surface of upper chamber were removed using cotton swabs, and cells that migrated to the bottom of the membrane were fixed and stained. The number of invaded cells was counted under a light microscope. To minimize the bias, five randomly selected fields with $200 \times$ magnification were counted, then the average number was calculated.

\section{RNA extraction, reverse transcription, and qRT-PCR analysis}

Total RNA was isolated from 252 gastric cancer samples using TRIzol@ reagent (15596-026, Invitrogen). A PrimeScript ${ }^{\mathrm{TM}}$ RT Master Mix (Perfect Real Time) kit (RR036A, Takara) was used to synthesize first-strand cDNA from total RNA. After, SYBR Green real-time PCR assays were performed using an ABI 7900HT (Applied Biosystems, USA). The expression level of RNA was normalized to the level of GAPDH. The primers for RT-PCR analysis were synthesized by Huagene (Shanghai, People's Republic of China). Primers were as follows: GAPDH-F, 5'-GCACCGTCAAGGCTGAGAAC-3', GAPDH-R, 5'-TGGTGAAGACGCCAGTGGA-3'; KLF7-F, 5'-CTCGGCAGTGGACATCTTG-3', KLF7-R, 5'-CACCAGTTTCAACGTCACCGT-3'.

\section{Western blotting}

KLF7 expression was assessed by Western blotting analysis, and the samples were normalized to GAPDH. Total proteins were extracted from the cultured cells solubilized in lysis buffer (RIPA Lysis Buffer, Thermo Scientific Pierce). The proteins were separated by sodium dodecyl sulfate-polyacrylamide gel electrophoresis and then transferred to polyvinylidene difluoride membranes (Bio-rad). The membranes were blocked with $5 \%$ bovine serum albumin (BSA) at room temperature for $2 \mathrm{~h}$ and incubated overnight at $4{ }^{\circ} \mathrm{C}$ with primary anti-KLF7 (1:1000, Ab197690, Abcam) and anti-GAPDH (1:5000, Santa Cruz). The membranes were subsequently washed and incubated with the appropriate secondary antibodies. After incubation with ECL, the protein bands were visualized.

\section{Immunohistochemistry (IHC) staining}

IHC staining was performed as described previously $[17,18]$. KLF7 anti-human rabbit antibody was used at a dilution of 1:100 (ab197690, Abcam); PBS was used as a negative control. Each section was 
evaluated and scored independently by two pathologists. A semi-quantitative scoring system was used in this assay $[19,20]$. The percentage of positively stained cells was scored as $0,<5 \% ; 1$, 5-25\%; 2, 26-50\%; 3, 51-75\%; and $4,>75 \%$. Intensity was scored as "-" (negative), "+” (weak), “++" (moderate) and "+++" (strong). We multiplied the percentage score by the staining intensity score to generate the immunoreactivity score (IS). High expression of KLF7 was defined as detectable immunoreactions in the nucleus with IS $\geq 4$.

\section{Statistical Analysis}

Two-tailed $\chi 2$ test was used to evaluate the expression difference between the clinicopathological features and KLF7 expression. The survival curves were estimated by Kaplan-Meier analysis, and $P$ values were calculated by log rank test. Univariate Cox proportional hazards regressions were applied to estimate the individual hazard ratio (HR) for the OS and RFS. The HR with a $95 \%$ confidence interval (CI) was measured to estimate the hazard risk of individual factors. All experiments were performed independently a minimum of three times. All $P$ values were two-sided, and $P<0.05$ was considered statistically significant. Statistical calculations were all performed using SPSS 17.0.
Table 2. Univariate and multivariate Cox proportional hazards analysis of KLF gene expression and overall survival for patients with gastric cancer in the TCGA cohort, Abbreviations: $\mathrm{CI}$, confidence interval; HR, hazard ratio, Bold type indicates statistical significance

\begin{tabular}{|c|c|c|c|c|}
\hline & \multicolumn{2}{|c|}{ Univariate analysis } & \multicolumn{2}{|c|}{ Multivariate analysis } \\
\hline Factor & HR $(95 \% \mathrm{CI})$ & $P$ & $\mathrm{HR}(95 \% \mathrm{CI})$ & $P$ \\
\hline Gender & $0.681(0.457-1.016)$ & 0.060 & & \\
\hline Age & $1.020(0.585-1.792)$ & 0.014 & $1.033(1.014-1.053)$ & 0.001 \\
\hline $\mathrm{T}$ category & $1.375(1.094-1.727)$ & 0.007 & $1.309(1.022-1.677)$ & 0.033 \\
\hline N stage & $1.300(1.113-1.518)$ & 0.001 & $1.240(1.053-1.461)$ & 0.010 \\
\hline M stage & $1.590(1.132-2.234)$ & 0.007 & $1.448(1.028-2.041)$ & 0.034 \\
\hline Grade & $1.313(0.933-1.848)$ & 0.119 & & \\
\hline Tumor location & $0.971(0.828-1.139)$ & 0.721 & & \\
\hline KLF1 & $1.389(0.455-4.241)$ & 0.564 & & \\
\hline KLF2 & $1.039(0.887-1.219)$ & 0.634 & & \\
\hline KLF3 & $0.807(0.583-1.117)$ & 0.196 & & \\
\hline KLF4 & $1.007(0.849-1.195)$ & 0.937 & & \\
\hline KLF5 & $0.900(0.781-1.037)$ & 0.145 & & \\
\hline KLF6 & $1.122(0.856-1.471)$ & 0.405 & & \\
\hline KLF7 & $1.353(1.089-1.683)$ & 0.006 & $1.293(1.002-1.672)$ & 0.049 \\
\hline KLF8 & $1.503(1.023-2.208)$ & 0.038 & $1.419(0.873-2.308)$ & 0.058 \\
\hline KLF9 & $1.148(0.964-1.367)$ & 0.120 & & \\
\hline KLF10 & $1.265(0.960-1.668)$ & 0.095 & & \\
\hline KLF11 & $1.212(0.901-1.629)$ & 0.203 & & \\
\hline KLF12 & $0.742(0.532-1.035)$ & 0.079 & & \\
\hline KLF13 & $0.694(0.413-1.164)$ & 0.167 & & \\
\hline KLF14 & $1.282(0.744-2.209)$ & 0.371 & & \\
\hline KLF15 & $1.023(0.835-1.253)$ & 0.826 & & \\
\hline KLF16 & $0.784(0.602-1.023)$ & 0.073 & & \\
\hline KLF17 & $1.041(0.606-1.787)$ & 0.884 & & \\
\hline
\end{tabular}

\section{Results}

Clinical factors in the TCGA and validation cohorts

There were 360 eligible patients enrolled in the study, and the median age was 65, ranging from 30 to 90 years old. Two hundred thirty-four $(65.0 \%)$ were male patients and $126(35.0 \%)$ were female patients. The majority of the patients $(91.1 \%)$ had no distant metastases. The characteristics of the samples are shown in Table 1. The median length of follow-up was 16 months (range, 1 month-124 months) and 226 patients died at the end of follow-up.

In the validation cohort, the median age was 59 (ranging 21 to 85 years old). One hundred thirty-six (53.9\%) were male and 116 (46.1\%) were female patients. Sixty-nine patients $(27.4 \%)$ had no lymph node metastases while the remaining had lymph node metastases. None of the patients had distant metastases. The expression levels of KLF7 in this cohort were nearly normally distributed (data not shown); therefore, we divided the cohort into low or high KLF7 expression groups according to the median expression level. The median follow-up time of this cohort was 32 months. Gender, age of diagnosis, TNM, and tumor grade are shown in Table 1. 
Fig. 1. KLF7 has higher expression in gastric cancer than adjacent normal tissues. (a) KLF7 expression was significantly upregulated in gastric cancer compared to adjacent normal controls $(\mathrm{P}<0.001)$. (b) Representative images of KLF7 expression in gastric cancer. As visualized at $200 \times$ and $400 \times$ magnifications (lower panels).

KLF7 was an independent prognostic factor for OS in the TCGA cohort

We first treated KLF family gene expression levels as continuous variables. In univariate Cox proportion hazard ratio analysis, age, tumor (T) stage, node (N) stage, metastasis (M) stage, KLF7, and KLF8 expression were significantly associated with prognosis in terms of OS in the TCGA cohorts $(P<0.05$, Table 2). Factors that were significant in univariate analyses were then incorporated into multivariate analysis. Multivariate analysis after adjustment for potential prognostic factors demonstrated that age $(\mathrm{HR}=1.033,95 \%$ CI 1.014-1.053, $P=0.001$ ), $T$ stage (HR=1.309, 95\% CI 1.022-1.677, $P=0.033)$, N stage (HR=1.240, 95\% CI 1.053-1.461, $P=0.010$ ), M stage $(\mathrm{HR}=1.448,95 \%$ CI 1.028-2.041, $P=0.034)$, and KLF7 (HR=1.293, 95\% CI 1.002-1.672, $\quad P=0.049$ ) were independent predictors of OS, but KLF8 lost its significance (HR=1.419, 95\% CI 0.873-2.308, $P=0.058$ ) (Table 2).

KLF7 expression patterns in the validation database

We first studied KLF7 mRNA expression in 30 paired GC tissues and their adjacent normal controls. As anticipated, the KLF7 mRNA expression levels were significantly higher in cancer tissues than their paired adjacent normal Table 3. Association between KLF7 expression and clinicopathological factors in gastric cancers

\begin{tabular}{|c|c|c|c|c|}
\hline \multirow{2}{*}{ Characteristics } & \multirow{2}{*}{ Total } & \multicolumn{2}{|c|}{ KLF7 expression } & \multirow{2}{*}{$P$ value } \\
\hline & & Low expression & High expression & \\
\hline Gender & & & & 0.706 \\
\hline Male & 136 & 86 & 50 & \\
\hline Female & 116 & 76 & 40 & \\
\hline Age & & & & 0.528 \\
\hline$\geq 60$ & 121 & 63 & 58 & \\
\hline$<60$ & 131 & 63 & 68 & \\
\hline Primary site & & & & 0.637 \\
\hline Antrum/Distal & 95 & 46 & 49 & \\
\hline Cardia/Proximal & 86 & 45 & 41 & \\
\hline Fundus/Body & 48 & 26 & 22 & \\
\hline $\begin{array}{l}\text { Gastroesophageal Junction } \\
\text { Unspecific }\end{array}$ & 23 & 9 & 14 & \\
\hline Histologic grade & & & & 0.784 \\
\hline $\mathrm{G} 1 / \mathrm{G} 2$ & 107 & 55 & 52 & \\
\hline G3 & 141 & 70 & 71 & \\
\hline T stage & & & & 0.013 \\
\hline $\mathrm{T} 1$ & 3 & 3 & 0 & \\
\hline $\mathrm{T} 2$ & 37 & 26 & 11 & \\
\hline T3 & 111 & 52 & 59 & \\
\hline $\mathrm{T} 4$ & 101 & 45 & 56 & \\
\hline $\mathrm{N}$ stage & & & & 0.022 \\
\hline No & 69 & 42 & 27 & \\
\hline N1 & 52 & 29 & 23 & \\
\hline N2 & 58 & 20 & 38 & \\
\hline N3 & 73 & 35 & 38 & \\
\hline Lymphovascular invasion & & & & 0.009 \\
\hline Negative & 199 & 108 & 91 & \\
\hline Positive & 53 & 18 & 35 & \\
\hline Perineural invasion & & & & 0.669 \\
\hline Negative & 185 & 94 & 91 & \\
\hline Positive & 67 & 32 & 35 & \\
\hline
\end{tabular}

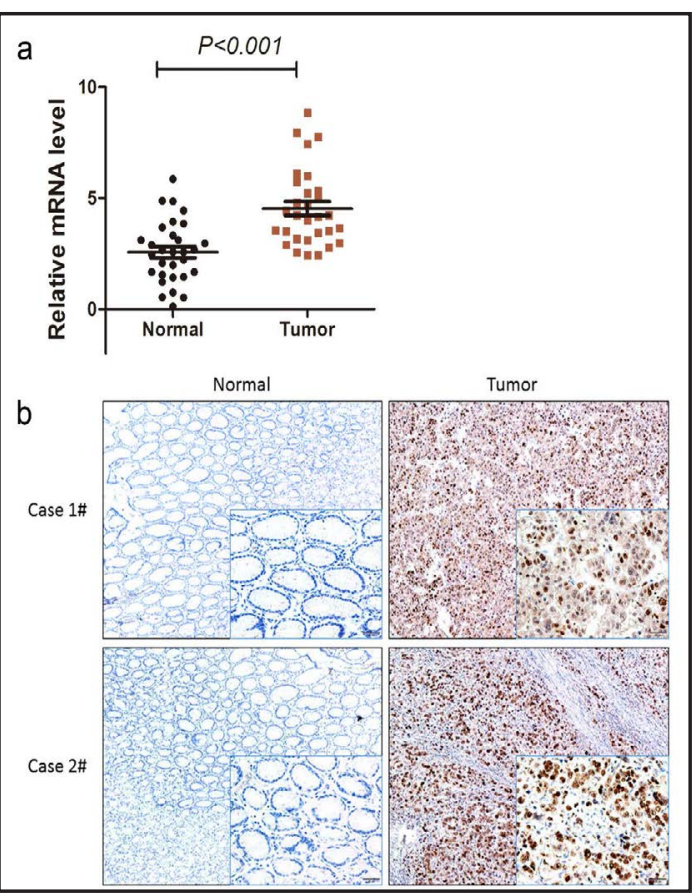
mucosa $(P<0.001$, Fig. 1a). Then, we tested KLF7 protein expression in those 30 paired cancer tissues and their normal tissues by IHC study, and the results further confirmed that KLF7 protein was expressed higher in cancer tissues than their normal controls (Fig. 1b). Specifically, we found the KLF7 mRNA expression was consistent with the protein expression levels. 

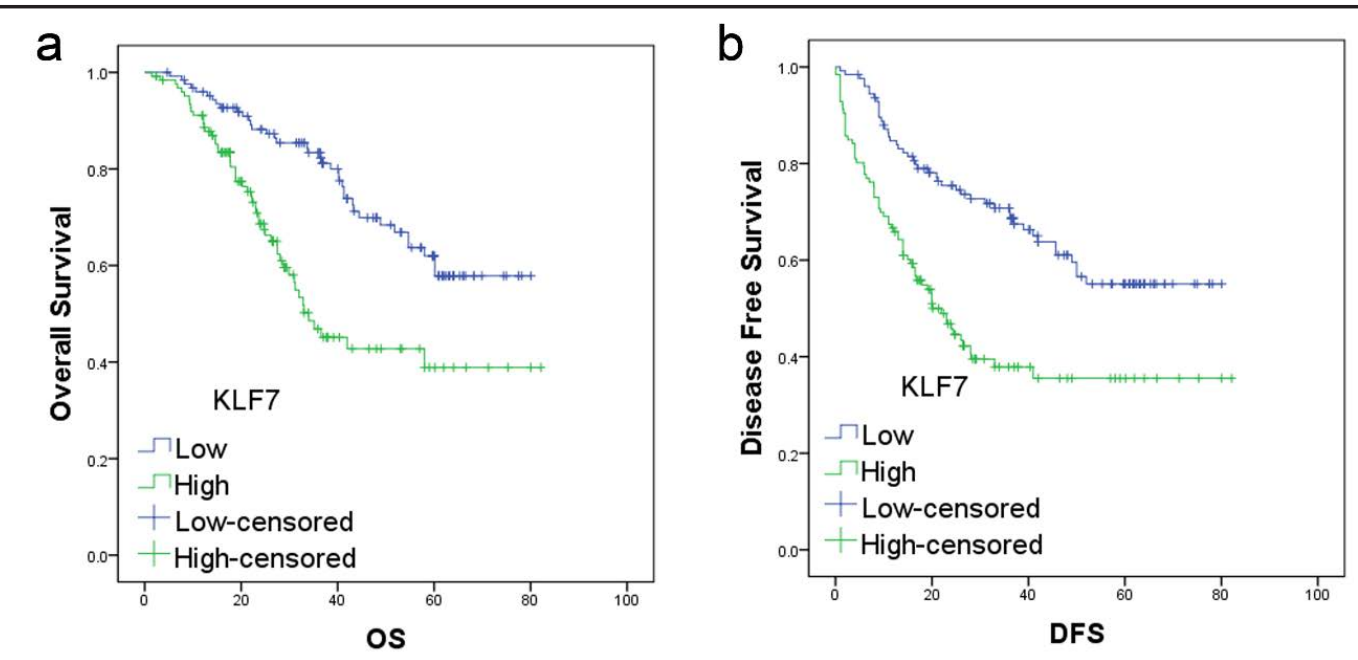

Fig. 2. Influence of KLF7 expression patterns on overall survival and disease free survival by Kaplan-Meier analyses in the validation cohort. (a) OS: $\chi^{2}=17.983, \mathrm{P}<0.001$; (b) DFS: $\chi^{2}=21.089, \mathrm{P}<0.001$.

Then, we studied KLF7 expression in 252 GC patients as described in Table 1. The patients were divided into high and low KLF7 mRNA expression subgroups according to median KLF7 expression value. Chi-square analysis demonstrated that high KLF7 expression was significantly correlated with $\mathrm{T}$ stage $(P=0.013), \quad \mathrm{N}$ stage $(P=0.022)$ and lymphovascular invasion $(\mathrm{P}=0.009)$ (Table 3).
Table 4. Univariate and multivariate Cox proportional hazards analysis of KLF gene expression and overall survival for patients with gastric cancer in the validation cohort, Abbreviations: CI, confidence interval; HR, hazard ratio, Bold type indicates statistical significance

\begin{tabular}{lcccc}
\hline \multirow{2}{*}{ Factor } & \multicolumn{2}{c}{ Univariate analysis } & \multicolumn{2}{c}{ Multivariate analysis } \\
& HR $(95 \% \mathrm{CI})$ & $\mathrm{P}$ & HR $(95 \% \mathrm{CI})$ & $\mathrm{P}$ \\
\hline Gender & $0.896(0.618-1.300)$ & 0.564 & & \\
Age & $1.183(0.817-1.713)$ & 0.373 & & \\
T category & $1.877(1.412-2.495)$ & $<0.001$ & $1.342(0.945-1.906)$ & 0.1 \\
N stage & $1.522(1.291-1.795)$ & 0.000 & $1.357(1.111-1.657)$ & 0.003 \\
Grade & $1.750(1.197-2.559)$ & 0.004 & $1.384(0.943-2.030)$ & 0.097 \\
Lymphovascular invasion & $1.540(1.039-2.282)$ & 0.032 & $1.639(1.090-2.465)$ & 0.018 \\
Perineural invasion & $2.208(1.474-3.308)$ & 0.000 & $1.702(1.122-2.580)$ & 0.012 \\
Tumor location & $1.059(0.898-1.249)$ & 0.496 & & \\
KLF7 & $1.847(1.264-2.700)$ & 0.002 & $1.669(1.138-2.448)$ & 0.009 \\
\hline
\end{tabular}

High KLF7 expression correlates with worse survival outcome in the validation cohort

To evaluate the correlation between KLF7 expression and 5-year patient survival, we constructed Kaplan-Meier survival curves using overall or disease free 5-year patient survival data to analyze the cases with high and low KLF7 expression. Our analysis revealed that overall survival (OS) in the high KLF7 expression subgroup was 38.9\% compared with $62.0 \%$ in the low expression subgroup. The log-rank analysis showed that the differences were significant $\left(\chi^{2}=17.983, P<0.001\right.$, Fig. 2a, Table 4). Similarly, patients with low KLF7 expression levels showed better 5-year DFS than those with high expression (55.1\% vs. 35.5\%, $\chi 2=21.089, \mathrm{P}<0.001$ ) (Fig. 2b), Table 5. Furthermore, multivariate Cox regression analysis showed that KLF7 expression is an independent factor for predicting both OS (HR: 1.669, 95\%CI: 1.138-2.448, P=0.009) and DFS (HR: 1.899, 95\%CI: 1.218-2.959, P=0.005) (Table 4 and 5). These data imply that KLF7 may be a potential prognostic marker in clinical practice. 
Fig. 3. Silencing KLF7 expression impaired gastric cancer cell migration. The knockdown effect of KLF7 in AGS and MGC803 cells after transfection of shRNA against KLF7 or scramble sequence was analyzed by Western blot (a) and RT-PCR (b). GAPDH was used as the loading control. (c) Knockdown of KLF7 expression does not change the cell proliferation of gastric cancer cell lines determined by CCK8 assay. (d) Knockdown of KLF7 expression impaired the migration of gastric cancer cells. The results are expressed as the mean \pm SD of three independent experiments $(* \mathrm{P}<0.05)$.

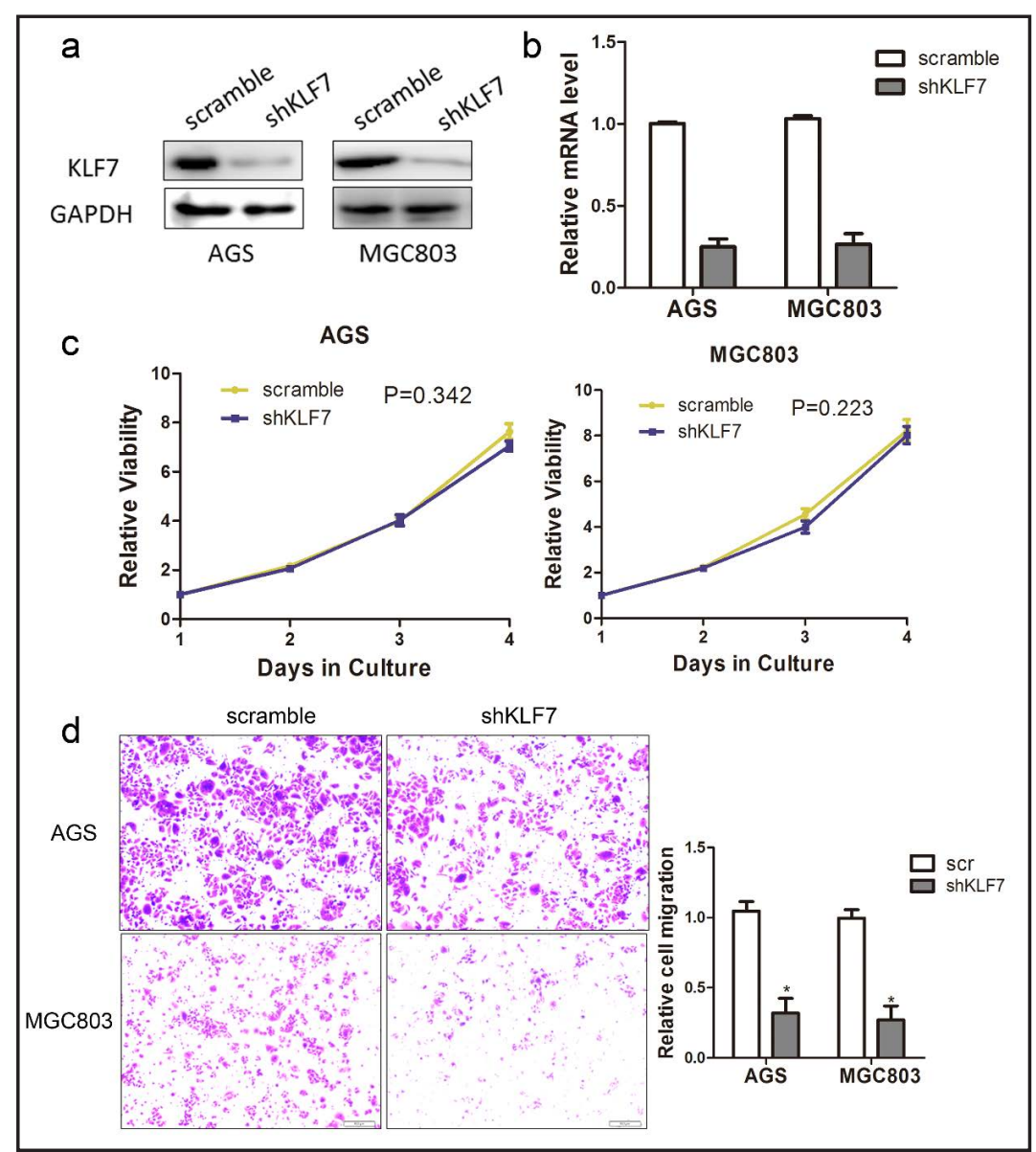

Silencing KLF7 expression inhibits the migration of human colon cancer cells

To determine the role of KLF7 in GC cell viability and progression, we used a lentivirus-mediated method to establish stable KLF7 knockdown in AGS and MGC803 cells, and the knockdown efficiency was determined by RT-PCR and Western blotting (Fig. $3 \mathrm{a}$ and $3 \mathrm{~b}$ ). A CCK8 assay showed that there was no significant difference in cell growth rates between KLF7-knockdown cells and their control cells. $(P>0.05$, and the results demonstrated that there was significantly decreased cell invasion abilities in KLF7 knockdown cells compared with control cells $(P<0.05$, Fig. 3d).

Table 5. Univariate and multivariate Cox proportional hazards analysis of KLF gene expression and disease-free survival for patients with gastric cancer in the validation cohort, Abbreviations: CI, confidence interval; HR, hazard ratio, Bold type indicates statistical significance

\begin{tabular}{lcccc}
\hline \multirow{2}{*}{ Factor } & \multicolumn{2}{c}{ Univariate analysis } & \multicolumn{2}{c}{ Multivariate analysis } \\
& HR $(95 \% \mathrm{CI})$ & $\mathrm{P}$ & HR $(95 \% \mathrm{CI})$ & $\mathrm{P}$ \\
\hline Gender & $0.819(0.535-1.253)$ & 0.357 & & \\
Age & $1.174(0.771-1.789)$ & 0.455 & & \\
T category & $1.808(1.317-2.480)$ & 0.000 & $1.178(0.783-1.774)$ & 0.431 \\
N stage & $1.627(1.342-1.973)$ & 0.000 & $1.480(1.160-1.889)$ & 0.002 \\
Grade & $1.598(1.042-2.450)$ & 0.032 & $1.241(0.805-1.913)$ & 0.327 \\
Lymphovascular invasion & $1.734(1.120-2.684)$ & 0.014 & $1.984(1.251-3.149)$ & 0.004 \\
Perineural invasion & $2.086(1.320-3.296)$ & 0.002 & $1.543(0.964-2.470)$ & 0.071 \\
Tumor location & $1.113(0.925-1.338)$ & 0.256 & & \\
KLF7 & $2.147(1.386-3.325)$ & 0.001 & $1.899(1.218-2.959)$ & 0.005
\end{tabular}




\section{Cellular Physiology Cell Physiol Biochem 2017;43:1090-1099 \begin{tabular}{ll|l} 
and Biochemistry Published online: October 05, 2017 & $\begin{array}{l}\text { (c) } 2017 \text { The Author(s). Published by S. Karger AG, Basel } \\
\text { www.karger.com/cpb }\end{array}$ \\
\hline
\end{tabular} \\ Jiang et al.: KLF7 in Gastric Cancer}

\section{Discussion}

Distant metastasis and local recurrence are the main reasons for failure of surgical cancer treatment. Currently, tumor-node-metastasis (TNM) stage is the most powerful predictor for survival in gastric cancer. However, the survival outcome is quite different, even for patients with the same TNM stage. Further understanding the biological mechanisms of the metastasis and progression of gastric cancer and developing effective measures to target this process are of vital importance. Much attention has been focused on the molecular-based prognostic markers, which are complementary to the data obtained by pathological diagnosis and can be used to provide more information for clinical practice. High-throughput RNA sequencing techniques have been widely used to identify reliable molecular biomarkers that may serve as new predictors or therapeutic targets. The TCGA database is a large-scale genomic database that primarily reports somatic changes of cancers including GC [21, 22]. The final goal of these tremendous efforts is to tailor patient treatment in a personalized manner and drive customization of disease care by implementing genetic tumor information. Importantly, the identified drivers can act as drug targets for disease amelioration, treatment biomarkers for patient categorization, and prognostic markers for patient survival [22]. Although the landscape of somatic aberrations in GC has been depicted, the utilization of relevant gene families in clinical situations has been limited.

The KLF7 protein is a member of the KLF transcription factors family that play important roles in regulating the expression of genes involved in cell growth, proliferation, differentiation and metabolism [23-25]. KLF7 CpG island methylation was correlated with diffuse sporadic gastric carcinogenesis but not intestinal GC [26]. In the present study, we first systematically studied the clinical significance of the KLF family in the TCGA database and found KLF7 was a significant prognostic factor for GC in both univariate and multivariate analysis. From 30 paired primary tumor and adjacent normal control, we found that the expression of KLF7 was significantly increased in primary colon cancer tumors compared with adjacent normal controls determined by both RT-PCR and IHC studies. In the validation cohort, high KLF7 expression was correlated with advanced $\mathrm{T}$ stage, $\mathrm{N}$ stage and lymphovascular invasion, which implied that KLF7 expression correlated with GC carcinogenesis and progression. Moreover, KLF7 mRNA levels were consistent with the protein levels as tested by RT-PCR and IHC study in the 30 paired GC samples. Specifically, the TCGA database lacks some important information, such as quality of surgery, sequential adjuvant therapy, etc. To reach a conclusion, we further validated another 252 GC patients, and the results demonstrated that higher KLF7 expression was significantly correlated with a shorter 5-year OS and DFS. These results further confirmed that KLF7 was an independent factor for survival in GC.

Cell proliferation and invasion are key steps for the metastatic progression of tumor cells [27]. Functional studies have found that reduced expression of KLF7 by shRNA significantly suppressed cell migration of AGS and MGC803 cells. These results further confirmed that KLF7 might participate in GC progression and metastasis.

A major strength of this study is that the information was obtained from two independent populations and in vitro study, but there are certain limitations. First, the prognosis of GC is affected by many factors, such as patients' social status, surgical techniques, and response to adjuvant therapy, so biomarkers from a single gene family are not sufficient. Second, no in vivo experiments using animals were performed to observe the effects of KLF7 on progression and metastases from mouse models. Thus, further in vivo animal studies are warranted to confirm our findings. Third, we mainly focus on the clinical significance of KLF7 in GC, so further studies are needed to explore the mechanism of KLF7 involvement in GC progression and metastases.

In conclusion, we found that KLF7 expression in GC tissues was significantly higher than in normal tissues. Moreover, KLF7 was an independent factor for survival in GC, and knockdown of KLF7 in GC cells suppressed cell migration. Hence, KLF7 is a new biomarker in GC. Inhibition of KLF7 expression in GC may represent a promising option to prevent local tumor advance and distant metastases. 


\section{Cellular Physiology Cell Physiol Biochem 2017;43:1090-1099 and Biochemistry \begin{tabular}{l|l} 
DOI: 10.1159/000481748 & Published 2017 The Author(s). Published by S. Karger AG, Basel \\
wwww.karger.com/cpb
\end{tabular}}

Jiang et al.: KLF7 in Gastric Cancer

\section{Disclosure Statement}

The authors declare no conflicts of interest.

\section{References}

$\rightarrow 1$ Siegel RL, Miller KD, Jemal A: Cancer Statistics, 2017. CA Cancer J Clin 2017;10.3322/caac.21387

-2 Chen W, Zheng R, Zhang S, Zhao P, Li G, Wu L, He J: The incidences and mortalities of major cancers in China, 2009. Chin J Cancer 2013;32:106-112.

-3 Gupta GP, Massague J: Cancer metastasis: building a framework. Cell 2006;127:679-695.

4 Li Q Wei P, Huang B, Xu Y, Li X, Li Y, Cai S, Li D: MAEL expression links epithelial-mesenchymal transition and stem cell properties in colorectal cancer. Int J Cancer 2016;139:2502-2511.

-5 Haldar SM, Ibrahim OA, Jain MK: Kruppel-like Factors (KLFs) in muscle biology. J Mol Cell Cardiol 2007;43:1-10.

6 Brey CW, Nelder MP, Hailemariam T, Gaugler R, Hashmi S: Kruppel-like family of transcription factors: an emerging new frontier in fat biology. Int J Biol Sci 2009;5:622-636.

-7 Pang CJ, Lemsaddek W, Alhashem YN, Bondzi C, Redmond LC, Ah-Son N, Dumur CI, Archer KJ, Haar JL, Lloyd JA, Trudel M: Kruppel-like factor 1 (KLF1), KLF2, and Myc control a regulatory network essential for embryonic erythropoiesis. Mol Cell Biol 2012;32:2628-2644.

-8 Sun F, He Z: Neuronal intrinsic barriers for axon regeneration in the adult CNS. Curr Opin Neurobiol 2010;20:510-518.

-9 Jang MK, Lee S, Jung MH: RNA-Seq Analysis Reveals a Negative Role of KLF16 in Adipogenesis. PLoS One 2016;11:e0162238.

10 Mukherjee D, Lu H, Yu L, He C, Lahiri SK, Li T, Zhao J: Kruppel-like factor 8 activates the transcription of C-X-C cytokine receptor type 4 to promote breast cancer cell invasion, transendothelial migration and metastasis. Oncotarget 2016; 7:23552-23568.

-11 Sun H, Peng Z, Tang H, Xie D, Jia Z, Zhong L, Zhao S, Ma Z, Gao Y, Zeng L, Luo R, Xie K: Loss of KLF4 and consequential downregulation of Smad7 exacerbate oncogenic TGF-beta signaling in and promote progression of hepatocellular carcinoma. Oncogene 2017;10.1038/onc.2016.447

12 Du C, Gao Y, Xu S, Jia J, Huang Z, Fan J, Wang X, He D, Guo P: KLF5 promotes cell migration by up-regulating FYN in bladder cancer cells. FEBS Lett 2016;590:408-418.

13 Cerami E, Gao J, Dogrusoz U, Gross BE, Sumer SO, Aksoy BA, Jacobsen A, Byrne CJ, Heuer ML, Larsson E, Antipin Y, Reva B, Goldberg AP, Sander C, Schultz N: The cBio cancer genomics portal: an open platform for exploring multidimensional cancer genomics data. Cancer Discov 2012;2:401-404.

14 Gao J, Aksoy BA, Dogrusoz U, Dresdner G, Gross B, Sumer SO, Sun Y, Jacobsen A, Sinha R, Larsson E, Cerami E, Sander C, Schultz N: Integrative analysis of complex cancer genomics and clinical profiles using the cBioPortal. Sci Signal 2013;6:pl1.

15 Quail MA, Smith M, Coupland P, Otto TD, Harris SR, Connor TR, Bertoni A, Swerdlow HP, Gu Y: A tale of three next generation sequencing platforms: comparison of Ion Torrent, Pacific Biosciences and Illumina MiSeq sequencers. BMC Genomics 2012;13:341.

-16 Autio R, Kilpinen S, Saarela M, Kallioniemi O, Hautaniemi S, Astola J: Comparison of Affymetrix data normalization methods using 6, 926 experiments across five array generations. BMC Bioinformatics 2009;10 Suppl 1:S24.

17 Li D, Peng Z, Tang H, Wei P, Kong X, Yan D, Huang F, Li Q, Le X, Xie K: KLF4-mediated negative regulation of IFITM3 expression plays a critical role in colon cancer pathogenesis. Clin Cancer Res 2011;17:3558-3568.

18 Li Q Qin Y, Wei P, Lian P, Li Y, Xu Y, Li X, Li D, Cai S: Gas1 Inhibits Metastatic and Metabolic Phenotypes in Colorectal Carcinoma. Mol Cancer Res 2016;14:830-840.

19 Sinicrope FA, Ruan SB, Cleary KR, Stephens LC, Lee JJ, Levin B: bcl-2 and p53 oncoprotein expression during colorectal tumorigenesis. Cancer Res 1995;55:237-241.

20 Li Q Wu J, Wei P, Xu Y, Zhuo C, Wang Y, Li D, Cai S: Overexpression of forkhead Box C2 promotes tumor metastasis and indicates poor prognosis in colon cancer via regulating epithelial-mesenchymal transition. Am J Cancer Res 2015;5:2022-2034. 


\section{Cellular Physiology Cell Physiol Biochem 2017;43:1090-1099

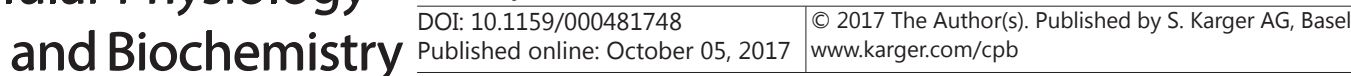

Jiang et al.: KLF7 in Gastric Cancer

-21 Cancer Genome Atlas Research N, Analysis Working Group: Asan U, Agency BCC, Brigham, Women's H, Broad I, Brown U, Case Western Reserve U, Dana-Farber Cancer I, Duke U, Greater Poland Cancer C, Harvard Medical S, Institute for Systems B, Leuven KU, Mayo C, Memorial Sloan Kettering Cancer C, National Cancer I, Nationwide Children's H, Stanford U, University of A, University of M, University of North C, University of P, University of R, University of Southern C, University of Texas MDACC, University of W, Van Andel Research I, Vanderbilt U, Washington U, Genome Sequencing Center: Broad I, Washington University in St L, Genome Characterization Centers BCCA, Broad I, Harvard Medical S, Sidney Kimmel Comprehensive Cancer Center at Johns Hopkins U, University of North C, University of Southern California Epigenome C, University of Texas MDACC, Van Andel Research I, Genome Data Analysis Centers: Broad I, Brown U, Harvard Medical S, Institute for Systems B, Memorial Sloan Kettering Cancer C, University of California Santa C, University of Texas MDACC, Biospecimen Core Resource: International Genomics C, Research Institute at Nationwide Children's H, Tissue Source Sites: Analytic Biologic S, Asan Medical C, Asterand B, Barretos Cancer H, BioreclamationIvt, Botkin Municipal C, Chonnam National University Medical S, Christiana Care Health S, Cureline, Duke U, Emory U, Erasmus U, Indiana University School of M, Institute of Oncology of M, International Genomics C, Invidumed, Israelitisches Krankenhaus H, Keimyung University School of M, Memorial Sloan Kettering Cancer C, National Cancer Center G, Ontario Tumour B, Peter MacCallum Cancer C, Pusan National University Medical S, Ribeirao Preto Medical S, St. Joseph's H, Medical C, St. Petersburg Academic U, Tayside Tissue B, University of D, University of Kansas Medical C, University of M, University of North Carolina at Chapel H, University of Pittsburgh School of M, University of Texas MDACC, Disease Working Group: Duke U, Memorial Sloan Kettering Cancer C, National Cancer I, University of Texas MDACC, Yonsei University College of M, Data Coordination Center CI, Project Team: National Institutes of H: Integrated genomic characterization of oesophageal carcinoma. Nature 2017;541:169-175.

-22 Wong HS, Juan YS, Wu MS, Zhang YF, Hsu YW, Chen HH, Liu WM, Chang WC: Integrative bioinformatic analyses of an oncogenomic profile reveal the biology of endometrial cancer and guide drug discovery. Oncotarget 2016; 7:5909-5923.

-23 Kanazawa A, Kawamura Y, Sekine A, Iida A, Tsunoda T, Kashiwagi A, Tanaka Y, Babazono T, Matsuda M, Kawai K, Iiizumi T, Fujioka T, Imanishi M, Kaku K, Iwamoto Y, Kawamori R, Kikkawa R, Nakamura Y, Maeda S: Single nucleotide polymorphisms in the gene encoding Kruppel-like factor 7 are associated with type 2 diabetes. Diabetologia 2005;48:1315-1322.

24 Zobel DP, Andreasen CH, Burgdorf KS, Andersson EA, Sandbaek A, Lauritzen T, Borch-Johnsen K, Jorgensen T, Maeda S, Nakamura Y, Eiberg H, Pedersen O, Hansen T: Variation in the gene encoding Kruppel-like factor 7 influences body fat: studies of 14818 Danes. Eur J Endocrinol 2009;160:603-609.

25 Lei L, Ma L, Nef S, Thai T, Parada LF: mKlf7, a potential transcriptional regulator of TrkA nerve growth factor receptor expression in sensory and sympathetic neurons. Development 2001;128:1147-1158.

26 Yang M, Kim HS, Cho MY: Different methylation profiles between intestinal and diffuse sporadic gastric carcinogenesis. Clin Res Hepatol Gastroenterol 2014;38:613-620.

27 Zhang Q, Chen X, Zhang X, Zhan J, Chen J: Knockdown of TMEM14A expression by RNAi inhibits the proliferation and invasion of human ovarian cancer cells. Biosci Rep 2016;36:e00298. 